\title{
PfHRP2 detection using plasmonic optrodes: performance analysis
}

\author{
Médéric Loyez ${ }^{1,3^{*}} \mathbb{D}$, Mathilde Wells², Stéphanie Hambÿe ${ }^{2}$, François Hubinon ${ }^{3}$, Bertrand Blankert ${ }^{2}$, \\ Ruddy Wattiez ${ }^{1}$ and Christophe Caucheteur ${ }^{3}$
}

\begin{abstract}
Background: Early malaria diagnosis and its profiling require the development of new sensing platforms enabling rapid and early analysis of parasites in blood or saliva, aside the widespread rapid diagnostic tests (RDTs).

Methods: This study shows the performance of a cost-effective optical fiber-based solution to target the presence of Plasmodium falciparum histidine-rich protein 2 (PfHRP2). Unclad multimode optical fiber probes are coated with a thin gold film to excite Surface Plasmon Resonance (SPR) yielding high sensitivity to bio-interactions between targets and bioreceptors grafted on the metal surface.

Results: Their performances are presented in laboratory conditions using PBS spiked with growing concentrations of purified target proteins and within in vitro cultures. Two probe configurations are studied through label-free detection and amplification using secondary antibodies to show the possibility to lower the intrisic limit of detection.

Conclusions: As malaria hits millions of people worldwide, the improvement and multiplexing of this optical fiber technique can be of great interest, especially for a future purpose of using multiple receptors on the fiber surface or several coated-nanoparticles as amplifiers.
\end{abstract}

Keywords: Optical fibers, Malaria diagnosis, Plasmodium falciparum, HRP2, LDH, SPR, Biosensing

\section{Background}

Malaria hits millions of people every year (228 million malaria cases worldwide in 2018) resulting in considerable number of deaths (405.000 in 2018), according to the World Health Organization (WHO) [1]. This parasitic disease is caused by several species of Plasmodium protozoa and can be transmitted by the bite of the female of more than thirty species of anopheline mosquitoes. The most common symptoms of Plasmodium spp infections are high fevers followed by chills and rigors by repeated cycles [2]. Severe cases can develop and lead to extreme anaemia, pulmonary, hepatic and cerebral forms. Some species can also stay dormant in the liver

*Correspondence: mederic.loyez@umons.ac.be

1 Proteomics and Microbiology Department, University of Mons, Champ de Mars 6, 7000 Mons, Belgium

Full list of author information is available at the end of the article upon inoculation and cause the recurrence of the disease. Malaria remains endemic in specific geographic areas, but thanks to the recent technological advances and large efforts invested in research and aid to developing countries, the hope for its eradication by 2030 becomes a real perspective [3].

Towards this malaria eradication, different and complementary paths need to be explored. For instance, the interruption of local transmissions is urgent because it is correlated with the rapid management of patients and the quality of care and treatment. This is often lacking in poor countries, and limits the decline in new infections. Diagnostics are usually performed using rapid diagnostic tests (RDTs) which are numerous and varied. Most of them rely on the detection of specific antigens released in blood or saliva by parasites. Some of them mainly focus on one species (such as Plasmodium falciparum which is responsible of more than $99 \%$ of cases in some regions),

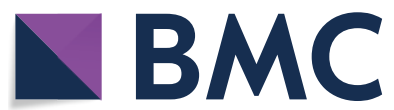

c) The Author(s) 2021. This article is licensed under a Creative Commons Attribution 4.0 International License, which permits use, sharing, adaptation, distribution and reproduction in any medium or format, as long as you give appropriate credit to the original author(s) and the source, provide a link to the Creative Commons licence, and indicate if changes were made. The images or other third party material in this article are included in the article's Creative Commons licence, unless indicated otherwise in a credit line to the material. If material is not included in the article's Creative Commons licence and your intended use is not permitted by statutory regulation or exceeds the permitted use, you will need to obtain permission directly from the copyright holder. To view a copy of this licence, visit http://creativecommons.org/licenses/by/4.0/. The Creative Commons Public Domain Dedication waiver (http://creativecommons.org/publicdomain/zero/1.0/) applies to the data made available in this article, unless otherwise stated in a credit line to the data. 
while others are multiplexed for the detection of other Plasmodium species (Plasmodium vivax, Plasmodium malariae and Plasmodium ovale). RDTs are mainly based on lateral flow immunochromatographic assays to detect free-circulating biomarkers[4]. They target histidine-rich protein 2 (PfHRP2) proteins using monoclonal antibodies, as HRP2 is present in abundance during the asexual cycle and in early gametocyte stages of $P$. falciparum parasites $[5,6]$. Other biosensors also target the lactate dehydrogenase $(p \mathrm{LDH})$. While PfHRP2 may remain detectable up to several days or even weeks after clearance of the disease, $p \mathrm{LDH}$ is a survival metabolic enzyme produced by the five species of parasites and does not persist after the infection has cleared. A combined detection of these two proteins could, therefore, be of interest to determine if a treatment is needed or if clearance already occurred $[7,8]$.

Among all the biosensors available to detect these proteins, immunosensors and especially paper-based dipsticks are probably the most spread tools, with several analytical benefits and cost efficiency [9]. They are based on a simple concept with low production costs, immediate readout, and remain the main solution for mass diagnostics on the field. However, they are often used late and their LOD (best-in-class RDTs are around $0.8 \mathrm{ng} / \mathrm{mL}$ for PfHRP2 or $\sim 200$ parasites $/ \mu \mathrm{L}$ ) does not always allow diagnostic at low parasitaemia levels, leading to false negative issues in very specific cases (early infections or asymptomatic cases) [10]. The effective threshold for the detection of biomarkers using RDTs is not straightforward to correlate with blood tests if not directly calibrated by the manufacturer, as the link between the HRP2 blood level and parasitaemia of infected patients is weak [11].

It is also important to mention that many studies call on microscopy for the quantification of malaria parasites in blood. Microscopy is a direct method for the detection of parasites, but it is user-dependent, poorly reproducible, and it often reveals false-positive results. There is, therefore, room for improvement in applied biosensing, as high-resolution systems are often limited to laboratory use and not compatible with low-cost portable platforms, which is of prime importance in remote places with little access to new technologies. This being said, RDTs are commercialized at large-scale and aim at diagnosing malaria directly on site. On the other hand, a growing interest on optical fiber (OF) sensors has arisen, and multiple research platforms have proven their interest to finely sense biomarkers at ultralow levels. Among all OF architectures available, unclad fibers remain the most practical platform towards a simple implementation for cost-effective and online monitoring. This configuration is, therefore, studied in this work.
Last but not least, a fair regulation between commercial diagnostic tests and availability of treatment and care is also needed. In fact, the race for the eradication of malaria faces with an increase in drug resistance (especially artemisinin resistance) due to unregulated treatment. It is, therefore, essential that patients are efficiently tested positively before taking anti-malarial drugs to avoid this phenomenon and allow current therapies to remain effective [12].

In this article, a lab-on-fiber platform for malaria diagnosis is studied. OF probes (optrodes) are unclad silica multimode fibers $(400 \mu \mathrm{m}$ core diametre). They present one centimetre-long gold-coated tips with roughly $50 \mathrm{~nm}$ of gold deposited on their outer surface to excite surface plasmons (SPR) in the visible wavelength range. They are connected to a portable white light source and a spectrometer for read-out. This configuration allows the acquisition of SPR spectra, that are analysed for further biological detection while bioreceptors are grafted on their surface. This method corresponds to the transposition of the commercially-available Kretschmann prism configuration to unclad OFs [13]. Specific structures can be implemented to excite SPR with OFs such as the use of Tilted Fiber Bragg Gratings (TFBGs) [14-17], Long Period Fiber Gratings (LPFGs) [18, 19], U-bent fibers $[20,21]$, and many other lab on fiber (LOF) architectures $[22,23]$. These probes have already proven their high potential for biomarkers detection in very different media, especially inside human tissues $[24,25]$ and body fluids [26, 27] among others [28, 29]. In this evolution to develop and evaluate new molecular detection techniques for malaria, the behaviour of our sensors is studied in different conditions to test their response and sensitivity against $p f \mathrm{HRP} 2$ biomarkers.

This original experimental study on Plasmodium falciparum cultures could open the door towards the integration of these OF probes into smaller biochips or bio-sticks connected to smartphone or specifically designed microfluidic packages. This could be of great interest for an innovative, portable and cost-effective diagnosis method [30-32].

\section{Methods \\ Materials}

Phosphate Buffer Saline (PBS) came from Thermo Fisher Scientific (Waltham, MA, USA). 6-mercapto-1-hexanol was purchased from Sigma-Aldrich (Merck, Darmstadt, Germany). The HRP2 proteins were purchased from Thermo Fischer Scientific. FT400UMT TECS Hard Clad, 0.39 NA, Step-index OF came from Thorlabs. Vytran CAC-400 compact fiber cleaver, the $400 \mu \mathrm{m}$ Y-bundle FC/PC and BFT1 connectors came from Thorlabs. Halogen white light source and HR4000 spectrometers came 
from OceanOptics. (Trimethylsilyls)-3-propanethiol came from VWR. Amine coupling kit (NHS/WSC, activation buffer and blocking BSA 1\%) came from Dojindo Molecular Technologies, Inc. (GERBU Biotechnik $\mathrm{GmbH}$, Germany). Laboratory-adapted P. falciparum strain 3D7 was purchased from BEI Resources (VA, USA). The RPMI 1640 powder supplemented with Hepes and L-Glutamine, as well as the AlbuMax ${ }^{\mathrm{TM}} \mathrm{II}$ and Gentamicin $(50 \mathrm{mg} / \mathrm{mL})$ were purchased from Thermo Fisher Scientific (Gibco ${ }^{\circledR}$, Waltham, MA, USA). Hypoxanthine and sodium bicarbonate were purchased from SigmaAldrich (Merck, Darmstadt, Germany). Concentrated red blood cells were provided by the Red Cross Organization (Namur, Belgium). ELISA kit for the detection and quantification of HRP2 (Ultra-Sensitive Quantimal Celisa KM8/KM8BP) came from Cellabs (Brookvale, Australia).

\section{Optical fibers preparation}

The OF-SPR sensors tips were manufactured using FT400 Step-Index multimode fiber with a core diameter of $400 \mu \mathrm{m}$ (Thorlabs). Pieces of $15 \mathrm{~cm}$ OF were cut, and a $4 \mathrm{~cm}$ end was stripped using a clamp. The stripped part of the OF was then immersed in acetone to remove the TECS hard polymer cladding, and cleaned manually by a cloth soaked in acetone until total removal of cladding remains. OFs were then cleaved at the right angle using a Vytran compact fiber cleaver (Thorlabs), to obtain $1 \mathrm{~cm}$ unclad sensing areas, and cut on the other side to connect the fiber. Final probe lengths of about $5 \mathrm{~cm}$ were used to avoid fiber bending during measurements. OF tips were then cleaned into piranha solution $\left(\mathrm{H}_{2} \mathrm{SO}_{4}\right.$ and
$\mathrm{H}_{2} \mathrm{O}_{2}$ 3:1) and silanized in methanol using $1 \%$ solution of (trimethylsilyls)-3-propanethiol during $15 \mathrm{~min}$, at room temperature (RT). OFs were then dried overnight at RT and mounted on a holder for a single $50 \mathrm{~nm}$ gold sputter deposition, which was performed vertically (Fig. 1a, b). The fibers were then directly stored in cleaned Petri dishes on a holder to avoid any dusts contaminations of the fiber tip.

\section{Bio-functionalization}

The immobilization of antibodies on the OFs as bioreceptors against PfHRP2 was performed through covalent bonding (Fig. 1c). OFs were immersed during $16 \mathrm{~h}$ in closed chambers of mercaptoundecanoic acid $2 \mathrm{mM}$ in absolute ethanol at room temperature (RT). They were then washed in absolute ethanol and placed into 125 $\mu \mathrm{L}$ vials of $50 \mathrm{mM}$ WSC and $50 \mathrm{mM}$ NHS in activation buffer during $10 \mathrm{~min}$ at RT. They were then washed with PBS and immersed during $1 \mathrm{~h} 30$ in anti-PfHRP2 antibodies. The blocking solution was then applied after another rinsing with PBS during 30 min at RT. Finally, the fibers were washed again in PBS and dried for biodetection experiments.

\section{Interrogation setup and data analyses}

OFs are connected through a bifurcator (Y-bundle FC/ $\mathrm{PC}$ ) to a white light source (halogen) and a spectrometer (Ocean Optics HR4000, with 500-900 nm range and resolution of $0.19 \mathrm{~nm}$ ). The spectrum released with this OFSPR technique results in a gaussian curve (Fig. 2) where the dip quality (full width at half maximum: FWHM and
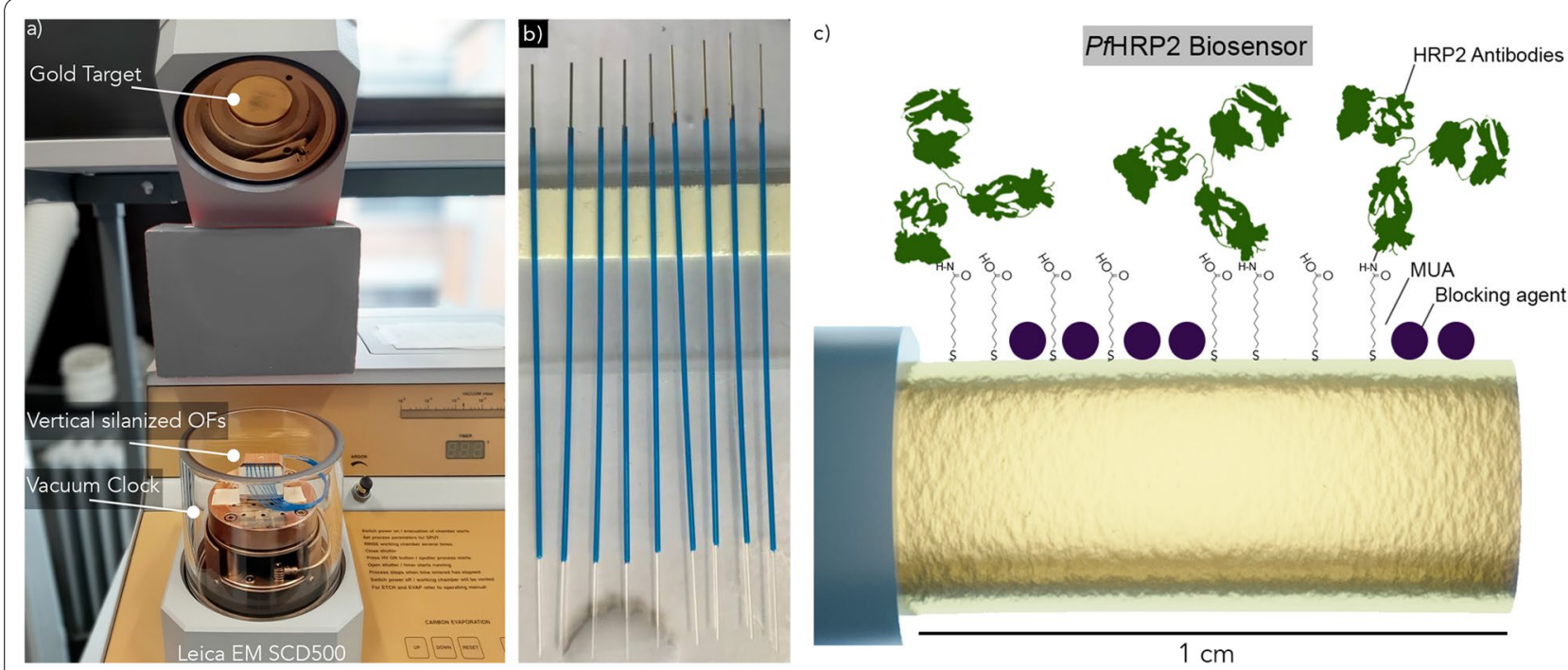

Fig. 1 a Picture of the gold sputter-coater. OFs are placed vertically inside the vacuum chamber. The quartz microbalance is at the level of the lower stage in the vacuum clock. b Picture of the prepared $400 \mu \mathrm{m}$ diametre OFs, ready for the functionalization process. $\mathbf{c}$ Scheme of the biochemical functionalization process with covalent coupling of HRP2 antibodies and implementation of the blocking agent 


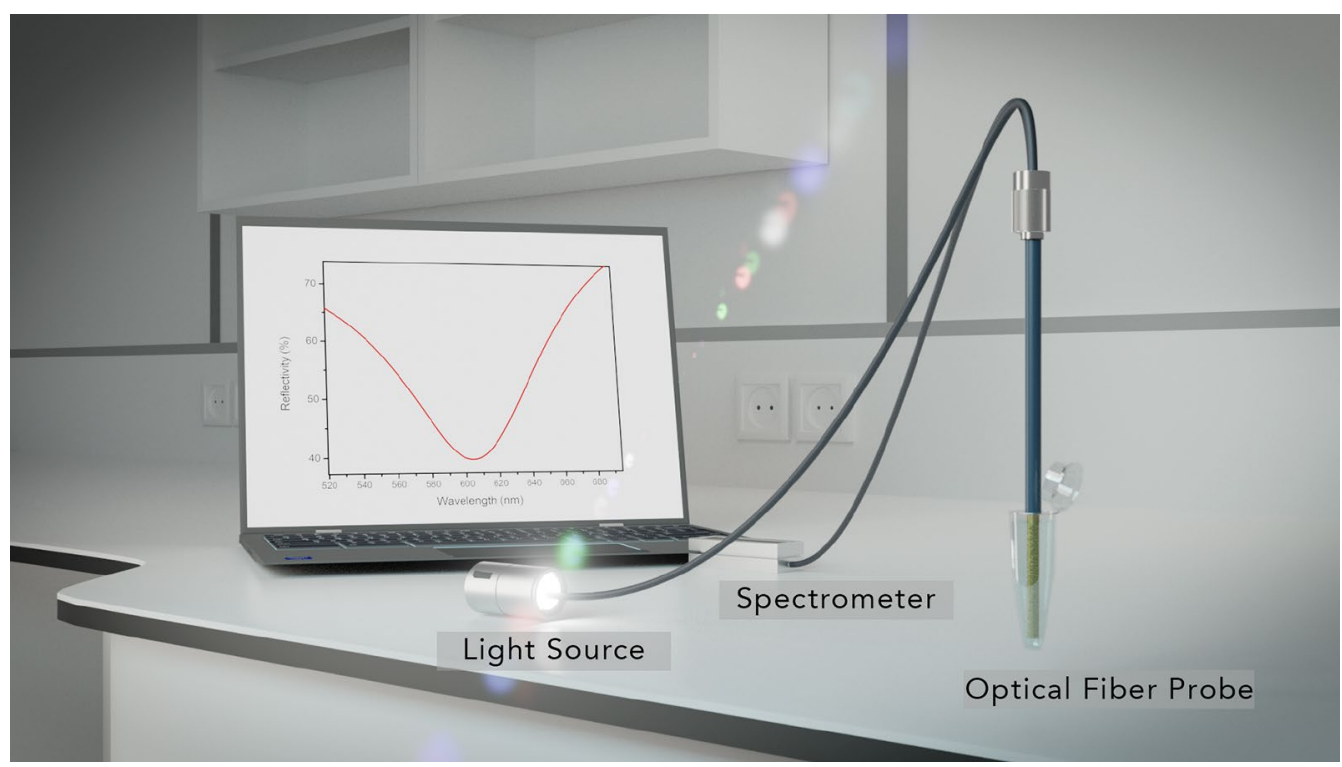

Fig. 2 Artistic view of the experimental setup with its plasmonic response

intensity: depth) is influenced by the mode propagation angle and the number of reflections occurring inside the OF [33]. Data were analysed through an in-house developed MATLAB program.

\section{Plasmodium falciparum cultures and sample preparation}

Laboratory-adapted strains 3D7 were maintained in continuous culture following the protocol proposed by Trager and Jensen with some modifications. Parasites were cultured in complete medium supplemented at 5\% haematocrit $\left(\mathrm{A} \mathrm{Rh}{ }^{+}\right)$. Briefly, complete medium was prepared from RPMI 1640 powder (25 mM Hepes, $2 \mathrm{mM}$ L-Glutamine) supplemented with $25 \mathrm{mM} \mathrm{NaHCO}, 0.5 \%$ AlbuMax $^{\mathrm{TM}}$ II, 2 mM Hypoxanthine and $0.002 \%$ Gentamicin. Cultures were incubated at $37{ }^{\circ} \mathrm{C}$ under humid atmosphere in a gas mixture containing $5 \% \mathrm{CO}_{2}$. Cultures were diluted with fresh red blood cells every other day [34].

For the purpose of sample preparation, cultures were synchronized $48 \mathrm{~h}$ prior to sample collecting to have a majority of ring-stage forms. The sorbitol method was used to achieve synchronicity. Both whole culture and culture supernatant were used for analysis. Briefly, whole cultures were incubated with a 5\% sorbitol solution at $37{ }^{\circ} \mathrm{C}$ for $15 \mathrm{~min}$. The lot then underwent successive centrifugation cycles and finally put back in continuous culture as described previously. Two days post synchronization, cultures were left to sediment for $90 \mathrm{~min}$ in the incubator. After this period, the supernatant was taken up without disturbing the red blood cell layer. The supernatant was centrifuged at $3260 \mathrm{~g}$ for $5 \mathrm{~min}$ to remove any red blood cell residues. Whole culture samples were taken up and did not undergo any specific treatment before being analysed. Parasitemia and ring-stage percentage were evaluated by optical microscopy (Additional file 1: 1). Blood smears were prepared for each culture and stained (Giemsa 10\%, × 1000). Parasitaemia was calculated as follows: [ number of infected red blood cells)/(total number of counted red blood cells)] $\times 100$. Percentage of ring-stage was determined as the [ number of ring-stage infected red blood cells)/(total number of infected red blood cells)] $\times 100$ [35].

\section{Validation using RDTs and ELISA}

The presence of HRP2 proteins in whole culture and supernatant was first qualitatively verified using rapid diagnostic tests before the OF experimentation (Malaria Ag Pf/Pan from Bioline). The HRP2 proteins spiked in PBS were also tested to verify their detection. These RDTs report a sensitivity of $99.7 \%$ and a specificity of 99.5\%. $5 \mu \mathrm{L}$ - sample was placed on the round well and diluted with four drops of the included assay diluent. The result was read after $15 \mathrm{~min}$, when the migration of the sample on the colored bands was completed. The color of the control line attests the good migration while the apparition of a second band attests the presence of HRP2 proteins. HRP2 quantification in cultures analysed by the OFs was performed afterwards through an ELISA. Two distinct calibration curves were established using the HRP2-purified protein spiked into the blank culture medium or the culture medium supplemented at $5 \%$ haematocrit at different concentrations. The samples 

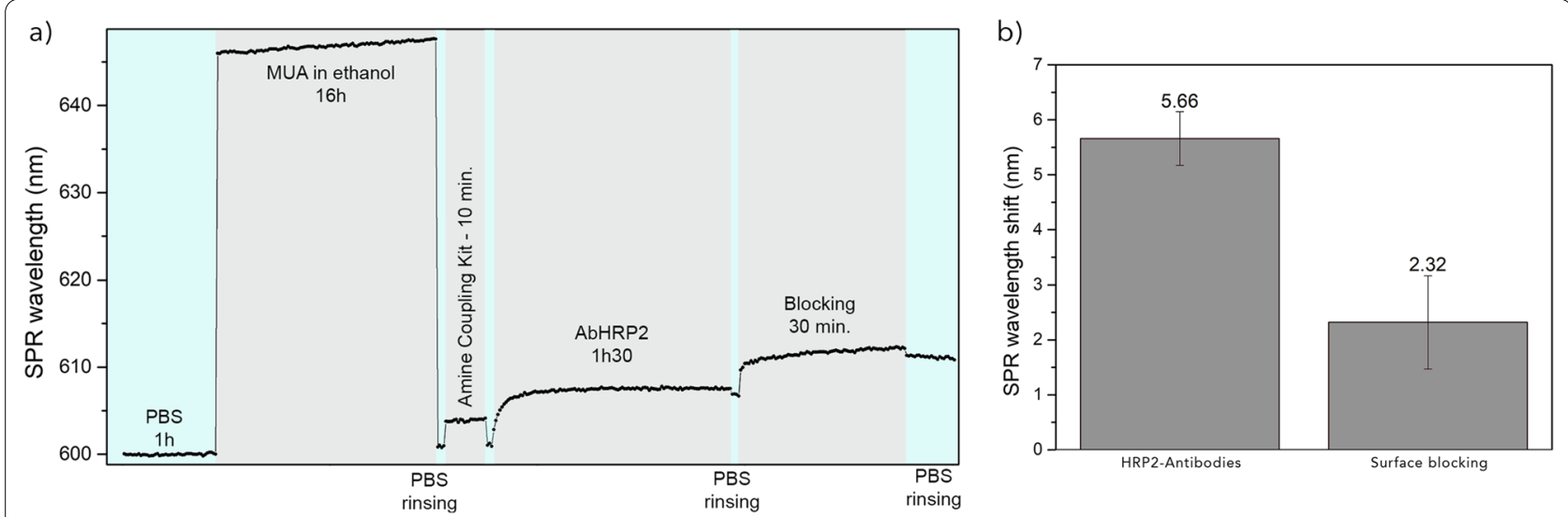

Fig. 3 a Sensorgram for the biofunctionalization in MUA, NHS/EDC, HRP2 antibodies, and casein blocking (shifts of the SPR-dip wavelength). b Mean \pm sd of the SPR wavelength shift obtained inside antibodies and blocking, after 90 and 30 min, respectively $(n=6)$

were diluted 1:1000 using culture medium or control blood and incubated on the microtitre plate in triplicates following the supplier recommendations.

\section{Results and discussion}

\section{Validation of the biosensor functionalization process}

Prior to biodetection experiments, the functionalization of the OFs and the related immobilization of the $p f \mathrm{HRP} 2$ antibodies was monitored in real time. First, the probe stability was verified into PBS during $1 \mathrm{~h}$. The fibers were then immersed overnight into MUA $2 \mathrm{mM}$ in ethanol to form a self-assembled monolayer (SAM) on the gold film. MUA molecules first bound to the surface and then selforganized, leading to a progressive shift of the SPR signal. The fibers were then rinsed and immersed into the solution of antibodies at a concentration of $20 \mu \mathrm{g} / \mathrm{mL}$ during 1h30. Finally, the blocking was also monitored (Fig. 3). This functionalization process shows the efficiency of the receptors binding to the fiber surface. This validation was performed for every lots of biosensors further tested. All these steps were performed in closed vials to avoid evaporation or perturbation of the medium during measurement, such as vibrations of the fiber probe.

\section{Optimization of the blocking process to work in complex matrices}

Live monitoring biosensors such as SPR-based platforms have to deal with non specific bindings occurring into complex media. Such unwanted interactions lead to false positive detections or block the interaction sites on the surface. To avoid these side effects, the blocking reaction was optimized by testing three well known methods, often used for immunoblotting or ELISA. The efficiency of three blocking agents was tested on top of gold-plated OFs using casein $0.1 \%$, fish gelatin $0.1 \%$ and BSA $1 \%$

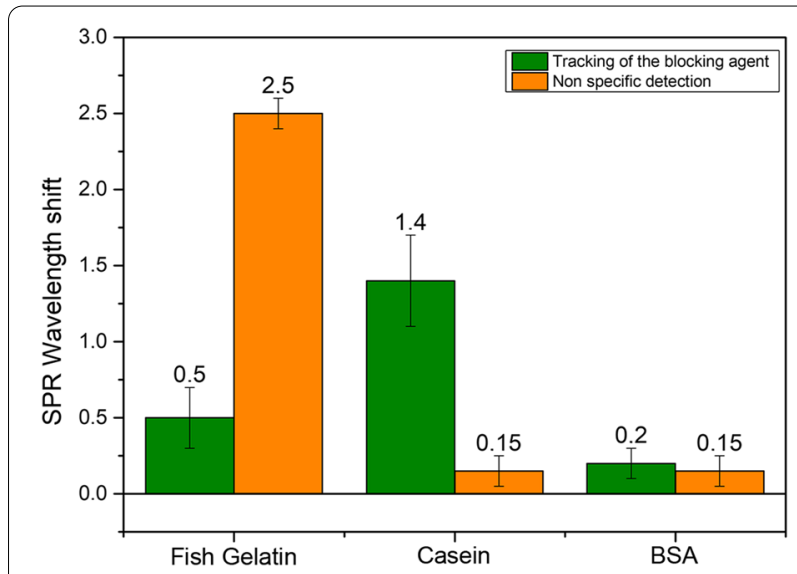

Fig. 4 Blocking process monitoring and efficiency evaluated by the SPR wavelength shift during blocking and immersion of the optical fibers into culture medium (mean $\pm s d, n=4$ different fibers tested for each condition)

$(\mathrm{m} / \mathrm{V})$, adapted from different protocols reported in the literature [36-38]. The fibers were functionalized using MUA, the amine-coupling kit, and blocked using these procedures. The sensors were then immersed in PBS to check the stability of the probes and verify the strong anchoring of the surface biolayer. Afterwards they were immersed in culture medium, where an efficient blocking should avoid any perturbations of the signal. The fibers were then rinsed and immersed back into PBS. The first measurements show that the fish gelatin is not sufficient enough to block the surface while BSA and casein present better performances. The casein blocking agent also leads to a better stability in both PBS and the culture medium (Fig. 4). That method was, therefore, selected for further biosensing analyses. The latter is also easier to track during the immobilization process than the BSA, probably because its anchoring on the fiber surface provokes 
a higher surface refractive index shift, due to molecular sizes and weight differences or by its organization on the probe (homogeneity, surface shape). The organization of the blocking layers onto the surface may play a crucial role for the process monitoring and for the protection against non-specific adsorption.

\section{Detection of spiked PfHRP2 using optial fiber biosensors}

OFs were immersed into PBS spiked with growing concentrations of PfHRP2. First, the fibers were placed into blank PBS and then into PBS + HRP2. The shift was monitored in real time and the final detection was considered 5 min after the immersion of the probe. These shifts are reported in Fig. 5a. The detection starts at around $1 \mu \mathrm{g} /$ $\mathrm{mL}$ in a label-free configuration. However, the needed LOD is often below that level, so that a sandwich bioassay should be foreseen. For instance, the use of coupled nanoparticles or secondary binders are often used to enhance the signal shift due to their higher effects on the surface refractive index. In this study, secondary antibodies were implemented to bind to the anchored HRP2 proteins in a sandwich-like assay. Using these amplifiers, the detection threshold was improved as shown on Fig. 5b where the signal shift after amplification begins at lower concentrations, which were not detected with the label-free configuration. Here, a significant plasmonic shift starts at around $100 \mathrm{ng} / \mathrm{mL}$.

\section{Detection of HRP2 in culture supernatant and whole culture}

The detection of HRP2 proteins was finally tested in vitro using cultures of $P$. falciparum. First, the optical fiber probes were immersed in PBS and then in cultures at 5\% Ht without parasites (control) to verify their specificity but also to determine its starting SPR wavelength, as it is driven by the effective refractive index of the solution. There was no significant red shift observed in the control medium within $10 \mathrm{~min}$. Its own refractive index is indeed close to the one of our PBS buffer which is equal to 1.3367 (given at $589 \mathrm{~nm}$ ). The plasmonic probes were then immersed back in PBS to clean their surface and were immersed in Plasmodium culture for $10 \mathrm{~min}$. A progressive red shift in the upper wavelengths of the SPR signal was observed, as presented in Fig. 6a. The fibers were then rinsed and disinfected into ethanol.

The detection of HRP2 inside these tested cultures was also attested by a strip-test showing the presence of the target by the apparition of a red band (Fig. 6b). An ELISA assay was also performed afterwards to quantify the amount of proteins in these cultures (Additional file 1:2). It appears that the whole cultures tested show a concentration of HRP2 often ranging around $1 \mu \mathrm{g} / \mathrm{mL}$ while its supernatant following centrifugation has a much lower HRP2 concentration. It also seems impossible to precisely link the parasitaemia to the HRP2 expression, as it is often pointed out in the literature. Depending on the culture-time and synchronization, both parasitaemia and concentrations of HRP2 can be highly impacted.

\section{Discussion of the performances and tunability of the lab-on-fiber technology}

Lab-on-fiber technology has shown major advances over the last decade $[39,40]$. The unclad fiber approach presented in this article also remains a practical method to make use of plasmonics inside low-volume
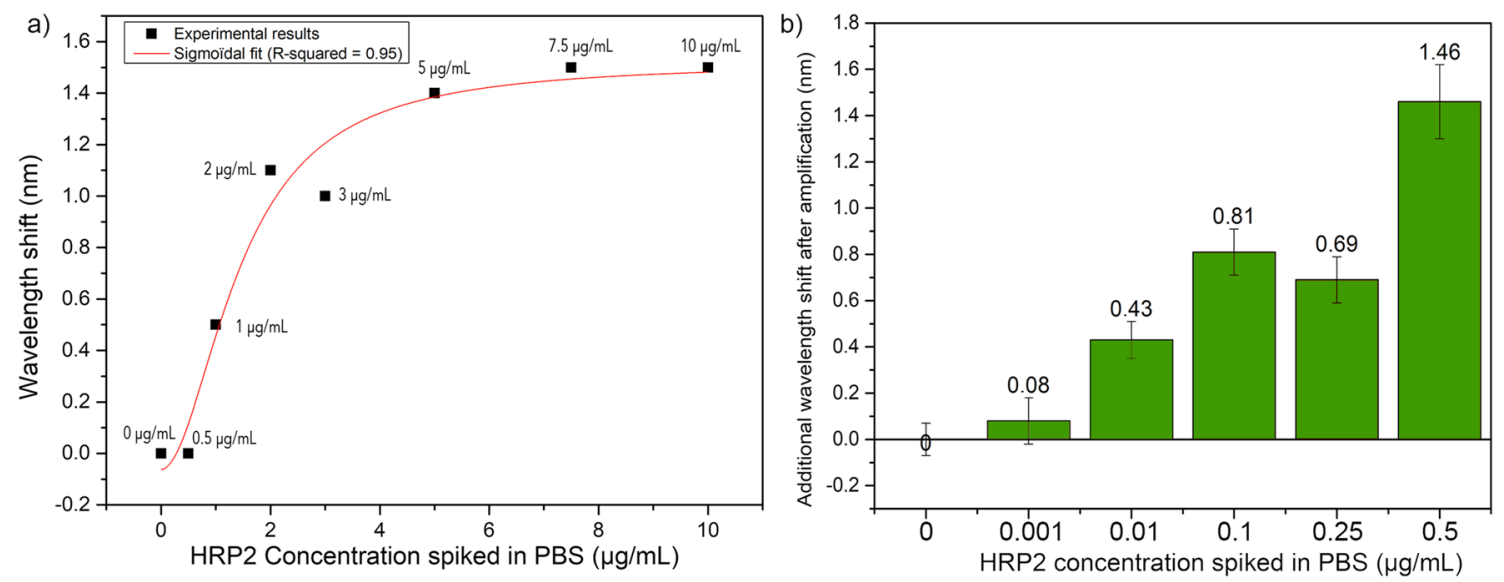

Fig. 5 a Label-free detection of HRP2 spiked in PBS at different concentrations (each experimental point represents the data obtained by a different optical fiber biosensor). $\mathbf{b}$ Shift observed after the addition of antibodies as amplification tags (mean $\pm \mathrm{sd}, \mathrm{n}=3 \mathrm{different}$ fibers for each concentration) 


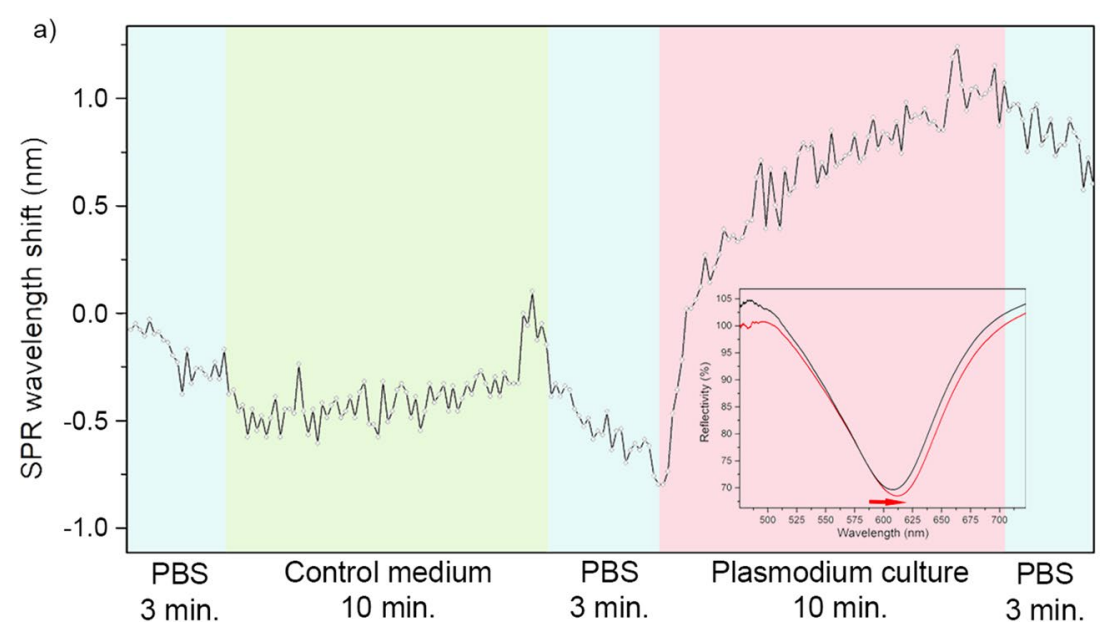

b)

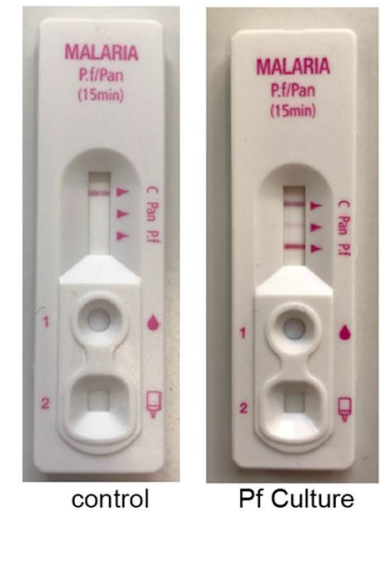

Fig. 6 a Sensorgram showing the live SPR response in both control medium and Plasmodium culture containing HRP2 proteins, b as verified by a commercial strip test

samples and to quicken the acquisition time compared to routine assays such as the ELISA [41]. It leads to highly tunable OFs that are interesting for many applications, especially to monitor live molecular events. In this paper, biofunctionalized optrodes were studied and optimized for the detection of HRP2 proteins in the framework of malaria detection. The possibility to use amplifiers to lower the LOD was shown, and the fibers were tested in complex media such as in vitro cultures of $P$. falciparum. The performances reached in this paper still leave room for improvements, especially to achieve more user-friendly interfaces. However, the many assets of OFs and their ease of implementation would enable substantial alternatives to the traditional equipment currently used at a research level, for instance, their combination with smartphones and portable devices, the elaboration of online bioprofiling databases [30].

The use of other molecular amplifiers to improve the LOD and reach the performances of best-in-class RDTs can also be investigated and optimized, such as the coupling of gold nanoparticles or nano-shell particles, often put in evidence in this field of research [42, 43]. The use of more specific targets such as $p \mathrm{LDH}$ or other bioreceptors, such as DNA aptamers or molecularly imprinted polymers (MIPs) could also be investigated to improve biosensing responses [44].

\section{Conclusion}

In this work, the detection of HRP2 proteins with unclad OF probes through plasmonic assays was performed. By anchoring antibodies on the fiber surface, it was possible to monitor the presence of the target proteins by a labelfree configuration (down to $1 \mu \mathrm{g} / \mathrm{mL}$ ) while its enhancement by the affinity of secondary binders can improve the sensor sensitivity by a factor of 10 . The detection was also performed on Plasmodium cultures, where HRP2 levels were tested by commercially available strip tests and a quantitative ELISA. As malaria affects many people around the world, improvement and multiplexing of this technique can be of great interest, especially because of its high tunability, both in terms of surface biochemistry and in terms of live spectral acquisition. The use of multiple receptors on the fiber surface or several coated-nanoparticles as amplifiers could still improve its LOD and lead to a practical tool, e.g. to discriminate the parasite causing malaria and early diagnosis purposes.

\section{Supplementary Information}

The online version contains supplementary material available at https://doi. org/10.1186/s12936-021-03863-3.

Additional file 1: 1. Optical microscopy of Plasmodium falciparum cultures. 2. Detection of PfHRP2 through ELISA.

\section{Acknowledgements}

We thank Magnetrap SA for funding this project and for the opportunity to work on these HRP2 biosensors. We thank Mariel David for her help during the preparation of the optical fiber probes.

\section{Authors' contributions}

ML: Optical fiber probes preparation, experiments, and writing. MW: Cultures and experiments, writing. SH: Cultures preparations. FH Optical fiber probes preparations and experiments, data analysis. BB and RW: Supervision. CC: Supervision and data analysis. All authors read and approved the final manuscript. 


\section{Funding}

This work was financially supported by Magnetrap (www.magnetrap.eu, Belgium), by the Fonds de la Recherche Scientifique (FNRS, Belgium) under the Postdoctoral Researcher grant (C.R.) of Médéric Loyez (FRS-FNRS) and the Associate Researcher Position of Christophe Caucheteur (FRS-FNRS).

\section{Availability of data and materials}

The dataset(s) supporting the conclusions of this article is(are) included within the article (and its additional file(s))

\section{Declarations}

\section{Ethics approval and consent to participate}

Not applicable.

\section{Consent for publication}

Not applicable.

\section{Competing interests}

The authors declare no competing interests.

\section{Author details}

${ }^{1}$ Proteomics and Microbiology Department, University of Mons, Champ de Mars 6, 7000 Mons, Belgium. ${ }^{2}$ Laboratory of Pharmaceutical Analysis, University of Mons, Avenue Maistriau 15, 7000 Mons, Belgium. ${ }^{3}$ Electromagnetism and Telecommunications Department, University of Mons, Bld. Dolez 31 , 7000 Mons, Belgium.

Received: 23 February 2021 Accepted: 23 July 2021

Published online: 28 July 2021

\section{References}

1. World Health Organization. World Malaria Report 2019. Geneva: World Health Organization; 2019.

2. Jain P, Chakma B, Patra S, Goswami P. Potential biomarkers and their applications for rapid and reliable detection of malaria. Biomed Res Int. 2014;2014:852645

3. Krampa FD, Aniweh Y, Kanyong P, Awandare GA. Recent advances in the development of biosensors for malaria diagnosis. Sensors (Switzerland). 2020:20:799.

4. Fung AO, Damoiseaux R, Grundeen S, Panes JL, Horton DH, Judy JW, et al. Quantitative detection of PfHRP2 in saliva of malaria patients in the Philippines. Malar J. 2012;11:175.

5. Lee N, Gatton ML, Pelecanos A, Bubb M, Gonzalez I, Bell D, et al. Identification of optimal epitopes for Plasmodium falciparum rapid diagnostic tests that target histidine-rich proteins 2 and 3. J Clin Microbiol. 2012;50:1397-405.

6. Kifude CM, Rajasekariah HG, Sullivan DJ, Stewart VA, Angov E, Martin SK, et al. Enzyme-linked immunosorbent assay for detection of Plasmodium falciparum histidine-rich protein 2 in blood, plasma, and serum. Clin Vaccine Immunol. 2008;15:1012-8.

7. Martiáñez-Vendrell X, Jiménez A, Vásquez A, Campillo A, Incardona S, González R, et al. Quantification of malaria antigens PfHRP2 and pLDH by quantitative suspension array technology in whole blood, dried blood spot and plasma. Malar J. 2020;19:12.

8. Deraney RN, Mace CR, Rolland JP, Schonhorn JE. Multiplexed, patterned-paper immunoassay for detection of malaria and dengue fever. Anal Chem. 2016;88:6161-5.

9. Andryukov BG, Besednova NN, Romashko RV, Zaporozhets TS, Efimov TA. Label-free biosensors for laboratory-based diagnostics of infections: current achievements and new trends. Biosensors. 2020;10:11.

10. Jimenez A, Rees-Channer RR, Perera R, Gamboa D, Chiodini PL, González IJ, et al. Analytical sensitivity of current best-in-class malaria rapid diagnostic tests. Malar J. 2017;16:128.
11. Pava Z, Echeverry DF, Díaz G, Murillo C. Short report: large variation in detection of histidine-rich protein 2 in Plasmodium falciparum isolates from Colombia. Am J Trop Med Hyg. 2010;83:834-7.

12. Mishra M, Mishra VK, Kashaw V, Iyer AK, Kashaw SK. Comprehensive review on various strategies for antimalarial drug discovery. Eur J Med Chem. 2017;125:1300-20

13. Lu J, Van Stappen T, Spasic D, Delport F, Vermeire S, Gils A, et al. Fiber optic-SPR platform for fast and sensitive infliximab detection in serum of inflammatory bowel disease patients. Biosens Bioelectron. 2016;79:173-9.

14. Albert J, Shao L, Caucheteur C. Tilted fiber Bragg grating sensors. Laser Photonics Rev. 2013;7:83-108.

15. Loyez M, Hassan EM, Lobry M, Liu F, Caucheteur C, Wattiez R, et al. Rapid detection of circulating breast cancer cells using a multiresonant optical fiber aptasensor with plasmonic amplification. ACS Sensors. 2020;5:454-63.

16. Lobry M, Loyez M, Hassan EM, Chah K, DeRosa MC, Goormaghtigh E, et al. Multimodal plasmonic optical fiber grating aptasensor. Opt Express. 2020;28:7539.

17. Lobry M, Loyez M, Chah K, Hassan EM, Goormaghtigh E, DeRosa MC, et al. HER2 biosensing through SPR-envelope tracking in plasmonic optical fiber gratings. Biomed Opt Express. 2020;11:4862-71.

18. Chiavaioli F, Baldini F, Tombelli S, Trono C, Giannetti A. Biosensing with optical fiber gratings. Nanophotonics. 2017;6:663-79.

19. Bandyopadhyay S, Biswas P, Chiavaioli F, Dey TK, Basumallick N, Trono C, et al. Long-period fiber grating: a specific design for biosensing applications. Appl Opt. 2017;56:9846.

20. Christopher C, Subrahmanyam A, Sai WR. Gold sputtered u-bent plastic optical fiber probes as SPR- and LSPR-based compact plasmonic sensors. Plasmonics. 2018:13:493-502.

21. Manoharan H, Kalita P, Gupta S, Sai WVR. Plasmonic biosensors for bacterial endotoxin detection on biomimetic C -18 supported fiber optic probes. Biosens Bioelectron. 2019;129:79-86.

22. Zubiate $P$, Urrutia A, Zamarreño CR, Egea-Urra J, Fernández-Irigoyen J, Giannetti A, et al. Fiber-based early diagnosis of venous thromboembolic disease by label-free D-dimer detection. Biosens Bioelectron X. 2019:2:100026.

23. Mowbray SE, Amiri AM. A brief overview of medical fiber optic biosensors and techniques in the modification for enhanced sensing ability. Diagnostics. 2019;9:23

24. Loyez M, Larrieu J, Chevineau S, Remmelink M, Leduc D, Bondue B, et al. In situ cancer diagnosis through online plasmonics. Biosens Bioelectron. 2019:131:104-12.

25. Ribaut C, Loyez M, Larrieu J, Chevineau S, Lambert P, Remmelink M, et al. Cancer biomarker sensing using packaged plasmonic optical fiber gratings : towards in vivo diagnosis. Biosens Bioelectron. 2017;92:449-56.

26. Han L, Guo T, Xie C, Xu P, Lao J, Zhang X, et al. Specific detection of aquaporin-2 using plasmonic tilted fiber grating sensors. J Light Technol. 2017;35:3360-5.

27. Qu JH, Dillen A, Saeys W, Lammertyn J, Spasic D. Advancements in SPR biosensing technology: an overview of recent trends in smart layers design, multiplexing concepts, continuous monitoring and in vivo sensing. Anal Chim Acta. 2019;1104:10-27.

28. Guo T, González-vila Á, Loyez M, Caucheteur C. Plasmonic optical fiber grating immunosensing : a review. Sensors (Basel). 2017;17:2732.

29. Leitao C, Pereira SO, Alberto N, Lobry M, Loyez M, Costa FM, et al. Cortisol in-fiber ultrasensitive plasmonic immunosensing. IEEE Sens J. 2020;21:3028-34

30. Sanjay M, Singh NK, Ngashangva L, Goswami P. A smartphone-based fiber-optic aptasensor for label-free detection of: Plasmodium falciparum glutamate dehydrogenase. Anal Methods. 2020;12:1333-41.

31. Lillehoj P, Huang M-C, Truong N, Ho C-M. Rapid electrochemical detection on a mobile phone. Lab Chip. 2013:15:4491-8.

32. Bremer K, Roth B. Fibre optic surface plasmon resonance sensor system designed for smartphones. Opt Express. 2015;23:17179.

33. Loyez M, Lobry M, Hassan EM, DeRosa MC, Caucheteur C, Wattiez R. HER2 breast cancer biomarker detection using a sandwich optical fiber assay. Talanta. 2021;221:121452 
34. Trigg PI. Invasion of erythrocytes by Plasmodium falciparum in vitro. Parasitology. 1975;71:433-6.

35. Trager W. Cultivation of malaria parasites. Methods Cell Biol. 1995;45:7-26.

36. Vogt RV, Phillips DL, Omar Henderson L, Whitfield W, Spierto FW. Quantitative differences among various proteins as blocking agents for ELISA microtiter plates. J Immunol Methods. 1987;101:43-50.

37. Reimhult K, Petersson K, Krozer A. QCM-D analysis of the performance of blocking agents on gold and polystyrene surfaces. Langmuir. 2008:24:8695-700.

38. Riquelme MV, Zhao H, Srinivasaraghavan V, Pruden A, Vikesland P, Agah M Optimizing blocking of nonspecific bacterial attachment to impedimetric biosensors. Sens Bio-Sensing Res. 2016;8:47-54.

39. Chiavaioli F. Recent development of resonance-based optical sensors and biosensors. Optics. 2020;1:255-8.

40. Sharma AK, Pandey AK, Kaur B. A Review of advancements (2007-2017) in plasmonics-based optical fiber sensors. Opt Fiber Technol. 2018;43:20-34

41. Horta S, Qu J-H, Dekimpe C, Bonnez Q, Vandenbulcke A, Tellier E, et al. Co(III)-NTA mediated antigen immobilization on a fiber optic-SPR biosensor for detection of autoantibodies in autoimmune diseases: application in immune-mediated thrombotic thrombocytopenic purpura. Anal Chem. 2020;92:13880-7.

42. Peeters B, Safdar S, Daems D, Goos P, Spasic D, Lammertyn J. Solid-phase PCR-amplified DNAzyme activity for real-time FO-SPR detection of the MCR-2 gene. Anal Chem. 2020;92:10783-91.

43. Daems D, Peeters B, Delport F, Remans T, Lammertyn J, Spasic D. Identification and quantification of celery allergens using fiber optic surface plasmon resonance PCR. Sensors (Switzerland). 2017;17:1754

44. Monfared YE. Overview of recent advances in the design of plasmonic fiber-optic biosensors. Biosensors (Basel). 2020;10:77.

\section{Publisher's Note}

Springer Nature remains neutral with regard to jurisdictional claims in published maps and institutional affiliations.
Ready to submit your research? Choose BMC and benefit from:

- fast, convenient online submission

- thorough peer review by experienced researchers in your field

- rapid publication on acceptance

- support for research data, including large and complex data types

- gold Open Access which fosters wider collaboration and increased citations

- maximum visibility for your research: over $100 \mathrm{M}$ website views per year

At BMC, research is always in progress.

Learn more biomedcentral.com/submissions 